\title{
Construction of Effective Minimal Active Microbial Consortia for Lignocellulose Degradation
}

\author{
Pilar Eliana Puentes-Téllez ${ }^{1,2}$. Joana Falcao Salles ${ }^{1}$
}

Received: 5 October 2017 / Accepted: 29 December 2017 / Published online: 1 February 2018

(C) The Author(s) 2018. This article is an open access publication

\begin{abstract}
Enriched microbial communities, obtained from environmental samples through selective processes, can effectively contribute to lignocellulose degradation. Unfortunately, fully controlled industrial degradation processes are difficult to reach given the intrinsically dynamic nature and complexity of the microbial communities, composed of a large number of culturable and unculturable species. The use of less complex but equally effective microbial consortia could improve their applications by allowing for more controlled industrial processes. Here, we combined ecological theory and enrichment principles to develop an effective lignocellulose-degrading minimal active microbial Consortia (MAMC). Following an enrichment of soil bacteria capable of degrading lignocellulose material from sugarcane origin, we applied a reductive-screening approach based on molecular phenotyping, identification, and metabolic characterization to obtain a selection of 18 lignocellulose-degrading strains representing four metabolic functional groups. We then generated 65 compositional replicates of MAMC containing five species each, which vary in the number of functional groups, metabolic potential, and degradation capacity. The characterization of the MAMC according to their degradation capacities and functional diversity measurements revealed that functional diversity positively correlated with the degradation of the most complex lignocellulosic fraction (lignin), indicating the importance of metabolic complementarity, whereas cellulose and hemicellulose degradation were either negatively or not affected by functional diversity. The screening method described here successfully led to the selection of effective MAMC, whose degradation potential reached up $96.5 \%$ of the degradation rates when all 18 species were present. A total of seven assembled synthetic communities were identified as the most effective MAMC. A consortium containing Stenotrophomonas maltophilia, Paenibacillus sp., Microbacterium sp., Chryseobacterium taiwanense, and Brevundimonas sp. was found to be the most effective degrading synthetic community.
\end{abstract}

Keywords Lignocellulose $\cdot$ Degradation $\cdot$ Microbial consortium $\cdot$ Functional diversity

Electronic supplementary material The online version of this article (https://doi.org/10.1007/s00248-017-1141-5) contains supplementary material, which is available to authorized users.

Joana Falcao Salles

j.falcao.salles@rug.nl

1 Microbial Community Ecology, GELIFES - Groningen Institute for Evolutionary Life Sciences, University of Groningen, Nijenborgh 7, 9747 AG Groningen, The Netherlands

2 Present address: Department of Biology, Institute of Environmental Biology, Ecology and Biodiversity Group, Padualaan 8, 3584 $\mathrm{CH}$ Utrecht, The Netherlands

\section{Introduction}

The biological degradation of lignocellulosic waste materials for subsequent energy production is considered a very promising and sustainable way to supply energy demands. For instance, lignocellulose agrowaste, such as straw and bagasse from sugarcane production, can potentially be used in industry, generating a wide range of value-added bioproducts (e.g., biogas, enzymes, antioxidants) and biofuels [1]. This degradation process relies on the breakdown of lignocellulosecomposed of hemicelluloses, cellulose, and the recalcitrant aromatic compound, lignin - through chemical, enzymatic, or thermomechanical processes that convert the polysaccharides into their constituent sugars [2]. 
The use of microbial consortia is considered an effective and sustainable way of promoting lignocellulose degradation, demonstrating enhanced degradation potential when compared to monoculture approaches, i.e., individual isolates [3-5]. Microbial consortia can be defined as communities ranging from two species communities to undefined, multispecies aggregations, where microorganisms use diverse mechanisms involving multiple complementary enzymes, particularly glycoside hydrolases (GHs), to deconstruct hemicellulose and cellulose. Lignin depolymerization on the other hand is achieved by using peroxidases and laccases [6]. Thus, the degradation of lignocellulose by microbial communities involves several complex and sometimes overlapping mechanisms, which are in general the result of a common effort by the members of the microbial community, due to resource complementarity.

Relationships among species diversity, stability, and function have been central topics in microbial ecology for several decades. Mounting evidence has demonstrated that the direct and positive relationship between function and diversity [7, 8] is often driven by the high complementarity between species, where higher productivity is observed in functionally diverse communities, as determined by their metabolic potential [9, 10]. In addition, ecological evidence supports the contention that microorganisms in a community depend on the activity of other microorganisms to grow, adapt, and reproduce [11-13]. To do so, they make use of complementary mechanisms involving the acquisition and exchange of metabolites to efficiently extract available energy resources. Thus, mixed populations are expected to perform functions that are difficult or even impossible for single species [14] while dealing with potential environmental fluctuations. These ecological principles underlie the higher effectiveness of lignocellulosedegrading microbial consortia and are currently being used to engineer microbial consortia able to effectively perform a range of processes [15].

The enrichment culture technique is a powerful tool to obtain microbial consortia with desired degradation properties $[16,17]$ because microbial consortia obtained with this method are closer to those functioning in nature [16]. Thus, in the last decades, several lignocellulose-degrading microbial consortia enriched from environmental samples have been identified and functionally characterized [18-22], revealing that functional redundancy is present in these systems [23]. Up to now, it is clear that microbial consortia production systems must account for the environmental relationships, distribution, abundance, and functional diversity of the participating members and their specific role during degradation [24].

Even though the analysis of enriched communities from environmental samples reveals the dominance of few bacterial phyla (e.g., Proteobacteria, Firmicutes, Chloroflexi, and Bacteroidetes), the number of ecological dominant species (culturable and unculturable) remains high, i.e., $>20$ identified strains [18, 25-29]. The complexity of such systems can make it difficult to disentangle the interactive network that ultimately drives the degradation process. Importantly, the lack of knowledge on the interactions between these large numbers of species during degradation hampers the upscaling of the consortia to an industrial system designed to obtain lignocellulose biodegradation and derived products like biofuels. In addition, the fact that not all effective strains from the enrichments can be isolated and grown in the lab limits their application for industrial purposes. Thus, designing an effective degrading consortium harboring a reduced number of culturable members could be advantageous, enabling a full characterization of the structural composition, the degradative power, and the interactive roles of degradation players. In other words, a better understanding of the degradation process could pave the way for a more efficient breakdown of lignocellulose for environmental and commercial purposes.

In order to obtain a minimal active microbial consortia (MAMC) from environmental samples, we used an ecologically driven, reductive-screening approach (reducing the number of species throughout the investigation), starting with the isolation of lignocellulose-degrading strains obtained by selection through an enrichment process of soil bacteria grown in sugarcane-biowaste lignocellulose substrates, followed by molecular phenotyping, identification, and metabolic characterization. Based on the metabolic characterization, 45 bacterial strains were classified as belonging to four functional metabolic groups. A set of 18 strain representatives of these groups were further used to construct a total of 65 synthetic communities containing five species each (65 compositional replicate MAMCs). Thus, MAMCs varied in their functional diversity, as determined by the number of functional metabolic groups, as well as in metabolic and degradation potential, while remaining constant in species richness. The MAMCs were evaluated for their degradation capacity, under the hypothesis that MAMC with higher functional diversity would lead to higher degradation rates.

\section{Materials and Methods}

\section{Isolation and Maintenance of Strains}

In order to obtain bacteria with high lignocellulose degradation capacity, we performed an enrichment experiment [28-30] using two sugarcane related substrates — bagasse (B) and straw ( $\mathrm{St}$ ) - and a soil inoculum obtained from a sugarcane plantation, which generated a total of 18 flasks including controls with substrate and no inoculum. Briefly, $10 \mathrm{~g}$ of soil inoculum was used to prepare a soil suspension by adding to $90 \mathrm{~mL}$ of sodium chloride $0.90 \%$ and $10 \mathrm{~g}$ of sterile gravel in $250-\mathrm{mL}$ flasks. After shaking for $1 \mathrm{~h}$ at $250 \mathrm{rpm}$ at room 
temperature $\left(20^{\circ} \mathrm{C}\right)$, aliquots of $250 \mu \mathrm{L}$ were inoculated to triplicate $100-\mathrm{mL}$ flasks containing $25 \mathrm{~mL}$ of mineral salt sterile medium (MSM) [30] with $1 \%$ of the lignocellulose sterile substrate. Cell density was controlled microscopically at regular time intervals and when cultures reached $10^{-9}$ cells/ $\mathrm{mL}$, an aliquot of $25 \mu \mathrm{L}$ of culture was transferred into $25 \mathrm{~mL}$ of fresh medium. After ten transfers and after the final flasks reached $10^{9}$ cells $/ \mathrm{mL}$, the enrichment process was stopped. A 2-mL sample from several flasks of lignocellulose-enriched soil community's final transfer (T10) was then used for colony isolation (for the enrichment experiment, see supplementary Fig. S1(a)). A homogenized sub-sample of $1 \mathrm{~mL}$ was used for serial dilutions in $\mathrm{NaCl} 0.85 \%$ until $10^{5}$ and plated onto minimal nutrient medium agar plates (Reasoner's 2A agar, R2A) (Becton Dickenson, Cockeysville, MD) in triplicates. Colonies were chosen according to morphological uniqueness via visual inspection and were further purified using several transfer steps and maintained in R2A agar. For long-term preservation and further studies, fresh biomass of the isolates obtained with LB broth ( $72 \mathrm{~h}$ of growth) was suspended in $20 \%$ glycerol and stored at $-20^{\circ} \mathrm{C}$.

\section{Genotypic Differentiation Using BOX-PCR (Molecular Fingerprinting)}

We performed colony BOX-PCR in order to compare the genomic profiles of all isolated colonies, and select for individual strains. BOX-PCR was performed with the primer A1R (CTACGGCAAGGCGACGCTGA) and the following PCR amplification conditions: $95^{\circ} \mathrm{C}$ for $2 \mathrm{~min} ; 35$ cycles of $94^{\circ} \mathrm{C}$ for $30 \mathrm{~s}, 50{ }^{\circ} \mathrm{C}$ for $1 \mathrm{~min}$, and $65^{\circ} \mathrm{C}$ for $8 \mathrm{~min}$; and a final extension step at $65{ }^{\circ} \mathrm{C}$ for $16 \mathrm{~min}$ [31]. PCR products were then run in $1.5 \%$ agarose gel and further analyzed (cluster analysis of BOX-PCR pattern) in GelCompar II (Applied Maths) using Dice coefficient and the UPGMA clustering. Isolates with a $>95 \%$ homology were assigned as belonging to the same strain.

\section{Phylogenetic Identification of Isolates by Partial 165 rRNA Gene Sequencing}

The DNA extraction from purified cultures of a total of 96 representative strains that belonged to unique BOX groups was performed with the UltraClean ${ }^{\circledR}$ Microbial DNA Isolation Kit (MoBio ${ }^{\circledR}$ Laboratories Inc., Carlsbad, CA, USA) according to the manufacturer's instructions. We assigned an ID number to each strain (ID strain number 1 to 96) for identification purposes during the study. Bacterial $16 \mathrm{~S}$ rRNA genes were amplified using primers B8F (5AGAGTTTGATCMTGGCTCAG-3' [32]) and U1406R (5'ACGGGCGGTGTGTRC-3' [33]). PCR reactions were done in a $50-\mu \mathrm{L}$ reaction mixture following the protocol of Taketani et al. [34]. PCR products were sequenced by Sanger technology (LGC Genomics, Germany). All resulting chromatograms were analyzed and trimmed for quality using the Lucy algorithm (http://rdp.cme.msu.edu/). Taxonomic assignment of the sequences (>99\% identity) was done using BLAST-N against the National Center for Biotechnology Information (NCBI) database (http://blast. ncbi.nlm.nih.gov/Blast.cgi) and confirmed at RDP (Ribosomal database project).

\section{Enzymatic Activities Related to Hemicellulose and Cellulose Degradation}

In order to determine distinct enzymatic degradation potentials of the strains identified by BOX-PCR and 16S rRNA gene sequencing, we quantified the enzymatic activities related to hemicellulose and cellulose breakdown during growth in MSM media with $1 \%$ of the specific lignocellulosic substrate from which they were originally obtained. Microbial cells plus substrate from 2-mL samples were harvested by centrifugation for $5 \mathrm{~min}$ at $10000 \mathrm{rpm}$ after $72 \mathrm{~h}$ of growth. The supernatant (secretome) was recovered and tested for enzymatic activity in triplicates using MUF- $\beta-D-x y l o p y r a n o s i d e$, MUF- $\beta$-D-mannopyranoside, MUF- $\beta$-D-galactopyranoside, MUF- $\beta$-D-cellobioside, and MUF- $\beta$-D-glucopyranoside as substrates. For the reaction, $10 \mu \mathrm{L}$ of MUF-substrate (10 $\mathrm{mM}$ in dimethyl sulfoxide), $15 \mu \mathrm{L}$ of Mcllvaine buffer (pH 6.8), and $25 \mu \mathrm{L}$ of each supernatant were mixed and incubated at $28^{\circ} \mathrm{C}$ for $45 \mathrm{~min}$ in the dark. The reaction was stopped by adding $150 \mu \mathrm{L}$ of $0.2 \mathrm{M}$ glycine- $\mathrm{NaOH}$ buffer (pH 10.4). Fluorescence was measured at an excitation of $365 \mathrm{~nm}$ and emission of $445 \mathrm{~nm}$. The negative controls consisted in sterile PCR water and a mixture without the MUF substrates. Enzyme activities were determined from the fluorescence units using a standard calibration curve built with glycine buffer ( $\mathrm{pH}$ 10.4) and expressed as rates of MUF production ( $\mu \mathrm{M}$ MUF per min at $28^{\circ} \mathrm{C} \mathrm{pH} 6.8$ ).

\section{Phenotypic Diversity (Biolog GEN III Plates)}

The metabolic profiles of 45 enzymatically active selected strains were monitored during growth on 71 energy sources of GEN III Biolog plates (Biolog Inc., Hayward, CA). The absorbance values at $590 \mathrm{~nm}$ were measured using a microplate reader (VersaMax microplate reader; Molecular Devices Corp.). For each strain, colonies obtained on R2A agar were pulled into inoculation fluid (IF-B) at an optical density $\left(\mathrm{OD}_{590}\right)$ of 0.03 . After $2 \mathrm{~h}$ of starvation in the IF-B fluid, $100 \mu \mathrm{L}$ was transferred to each well of the GEN III plates including one blank. The plates were incubated at $28^{\circ} \mathrm{C}$ and read every $12 \mathrm{~h}$ until $72 \mathrm{~h}$ of growth. The normalized sum of all measured time points for the well was used to calculate the area under the curve for each carbon source. These data were used to generate a principal component analysis (PCA) using 
Canoco v5.0 [35], which allowed the classification of strains into functional metabolic groups (FMG), by calculating the similarities in metabolic profiling across all 71 substrates.

\section{MAMC}

A total of 18 strains representing all FMG were used to create compositional replicates of five-species MAMC. Specifically, a total of 65 different synthetic communities were created by combining members of two, three, or all four FMG (Table 1), thus generating MAMC with different levels of functional diversity (two, three, or four FMG) but similar levels of species richness. For each level of functional diversity, we prepared three to four compositional replicates of each synthetic community, i.e., using the same number of species from the same FMG but different species, leading a total of 65 synthetic communities. The use of compositional replicates allowed for proper quantification of functional diversity on the substrate's degradation while controlling for the influence of species identity.

After selecting the composition of each one of the 65 MAMCs, these were constructed by mixing all strains in equal concentrations. Briefly, we grew the strains in R2A broth during $40 \mathrm{~h}\left(180 \mathrm{rpm}, 28^{\circ} \mathrm{C}\right)$ and adjusted each strain to an $\mathrm{OD}_{590}$ of 0.02 in $\mathrm{NaCl} 0.85 \%$. An aliquot of $125 \mu \mathrm{L}$ of each diluted strain (approximately $1 \times 10^{5}$ cells $/ \mathrm{mL}$, as determined by plate counting) was inoculated into $25 \mathrm{~mL}$ of MSM medium with $1 \%$ of straw (total cell concentration of $5 \times 10^{5}$ cells $/ \mathrm{mL}$ in $25 \mathrm{~mL}$ ). In order to generate a control with maximum functional diversity and species richness, we also created a synthetic community containing all selected strains (18 in total). For this, we inoculated approximately $3 \times 10^{4}$ cells $/ \mathrm{mL}$ of each strain. All consortia were incubated at $28{ }^{\circ} \mathrm{C} 180 \mathrm{rpm}$ for $96 \mathrm{~h}$.

\section{FTIR Analysis}

For the calibration set, pure cellulose (microcrystalline powder), hemicelluloses (xylan from birch wood), and lignin (hydrolytic) powders were obtained from Sigma-Aldrich Canada Ltd. (St. Louis MO) and were subsequently mixed in different proportions [36] to determine the relationship between their respective quantity in the synthetic community and representative Fourier transform infrared spectroscopy (FTIR) spectra [36]. Particle size of both the calibration set and samples was defined with a $106 \mu \mathrm{m}$ sieve. Spectra were recorded using a Perkin-Elmer VATR Two spectrometer (Waltham MA USA) in the wavenumber range of 800 $1800 \mathrm{~cm}^{-1}$ with a resolution of $4 \mathrm{~cm}^{-1}$ under ambient atmosphere at room temperature and in triplicates. The spectra were integrated and baseline corrected using the Spectrum TM software. The analysis was performed using the Unscrambler X (Camo Software Oslo Norway). A 5-point Savitzky-Golay smoothing algorithm was applied to the calibration set's spectra and used to predict concentrations in the samples (using partial least squares (PLS) regression). The predicted composition of each sample obtained with PLS was expressed as the percentage of degradation (from the initial amount; \%D) and was calculated for each lignocellulose fraction of the lignocellulose as follows: $\% \mathrm{D}=[(\mathrm{a}-\mathrm{b}) / \mathrm{a}] \times 100$; where $\mathrm{a}=$ percentage of the fraction in the substrate before incubation; $b=$ percentage of the fraction in the substrate after incubation.

\section{Functional Diversity Measurements}

The community niche $(\mathrm{CN})$ of a given MAMC was obtained based on the performance of each species on each one of the 71 carbon sources from the Biolog GEN III plate [9]. The CN value corresponds to the sum of the best performances per carbon source found in each synthetic community. Furthermore, we used the metabolic potential of each strain, also based on Biolog data, to calculate the functional attribute distance (FAD), used as a proxy of functional diversity [37, 38]. FAD was calculated by using Euclidean distance to calculate the pairwise distance between species (Function dist \{\} implemented in RStudio 1.0.136 [39].

\section{Results}

\section{Isolation and Identification of Enriched Bacterial Strains}

Samples from the end-point populations (Transfer 10) of all the cultures with substrate (including the controls) were diluted and plated onto R2A agar. A total of 157 isolates (up to 16 colonies per flask) were obtained from the last three plated dilutions. Molecular characterization of the purified strains using BOX PCR revealed a total of 96 unique groups across all samples, which were identified by sequencing of the $16 \mathrm{~S}$ rRNA gene in 72 bacterial species (see Table S1 supplementary information for identity and GeneBank accession numbers).

\section{Enzymatic Degradation Potentials}

The enzymatic degradation potential of 72 strains was measured after $72 \mathrm{~h}$ of growth under the enriched correspondent substrate. Results did not have a correlation with the substrate type in all MUF substrates $(p>0.05)$. The highest enzymatic activity in all secretomes was observed in the B-Dmannopyranoside activity (related to hemicellulose degradation) with an averaged $1.17 \mu \mathrm{M} \mathrm{MUF} / \mathrm{min}$ followed by $0.2 \mu \mathrm{M} \mathrm{MUF} / \mathrm{min}$ of $\beta$-D-cellobioside (involved in cellulose degradation) and $0.12 \mu \mathrm{M}$ MUF/min of B-D-xylopyranoside (as part of hemicellulolitic activities). Based on enzymatic 
Table 1 Description of the MAMC according to the number of functional metabolic groups. Type of mix according to the number of functional metabolic groups (FMG) combined (FMG I, FMG II, FMG III, FMG IV) and ID number of strains used in each synthetic community per functional group. All synthetic communities were at least three compositional replicates. m: identification of the synthetic community. For strain identification, see Table 2

\begin{tabular}{|c|c|c|c|c|c|c|c|c|c|c|c|c|c|}
\hline \multirow{3}{*}{\begin{tabular}{|c|}
$\begin{array}{c}\text { Type of } \\
\text { Mix }\end{array}$ \\
All 18 \\
\end{tabular}} & \multirow[b]{2}{*}{$\begin{array}{c}\text { Synthetic } \\
\text { community }\end{array}$} & \multicolumn{12}{|c|}{ FMG - Strains ID number } \\
\hline & & \multicolumn{3}{|c|}{$\mathbf{I}$} & \multicolumn{3}{|c|}{ II } & \multicolumn{3}{|c|}{ III } & \multicolumn{3}{|c|}{ IV } \\
\hline & $\mathrm{m} 1$ & & & & & & & & & & & & \\
\hline Type A: & $\mathrm{m} 2$ & 76 & 10 & & 49 & & & 68 & & & 41 & & \\
\hline Mixing & $\mathrm{m} 3$ & 76 & 26 & & 90 & & & 11 & & & 44 & & \\
\hline \multirow[t]{14}{*}{ four FMG } & $\mathrm{m} 4$ & 76 & 62 & & 57 & & & 29 & & & 25 & & \\
\hline & m5 & 10 & 62 & & 85 & & & 52 & & & 15 & & \\
\hline & m6 & 10 & & & 49 & 90 & & 11 & & & 25 & & \\
\hline & $\mathrm{m} 7$ & 62 & & & 49 & 57 & & 52 & & & 44 & & \\
\hline & $\mathrm{m} 8$ & 76 & & & 90 & 85 & & 18 & & & 41 & & \\
\hline & m9 & 10 & & & 49 & 85 & & 29 & & & 13 & & \\
\hline & $\mathrm{m} 10$ & 76 & & & 49 & & & 68 & 11 & & 41 & & \\
\hline & m11 & 10 & & & 90 & & & 68 & 29 & & 25 & & \\
\hline & $\mathrm{m} 12$ & 26 & & & 57 & & & 68 & 52 & & 44 & & \\
\hline & $\mathrm{m} 13$ & 62 & & & 85 & & & 29 & 18 & & 15 & & \\
\hline & m14 & 10 & & & 85 & & & 68 & & & 41 & 44 & \\
\hline & m15 & 26 & & & 90 & & & 11 & & & 41 & 25 & \\
\hline & $\mathrm{m} 16$ & 76 & & & 49 & & & 29 & & & 44 & 15 & \\
\hline & m17 & 62 & & & 57 & & & 52 & & & 13 & 41 & \\
\hline \multirow{36}{*}{$\begin{array}{l}\text { Type B: } \\
\text { mixing } \\
\text { two FMG }\end{array}$} & $\mathrm{m} 18$ & 76 & 10 & 62 & 49 & 57 & & & & & & & \\
\hline & m19 & 76 & 10 & 62 & 49 & 85 & & & & & & & \\
\hline & $\mathrm{m} 20$ & 76 & 10 & 62 & 57 & 85 & & & & & & & \\
\hline & $\mathrm{m} 21$ & 76 & 10 & & 49 & 57 & 85 & & & & & & \\
\hline & $\mathrm{m} 22$ & 76 & 62 & & 49 & 57 & 85 & & & & & & \\
\hline & $\mathrm{m} 23$ & 10 & 62 & & 49 & 57 & 85 & & & & & & \\
\hline & $\mathrm{m} 24$ & 76 & 10 & 62 & & & & 68 & 18 & & & & \\
\hline & $\mathrm{m} 25$ & 76 & 10 & 62 & & & & 68 & 29 & & & & \\
\hline & $\mathrm{m} 26$ & 76 & 10 & 62 & & & & 18 & 29 & & & & \\
\hline & $\mathrm{m} 27$ & 76 & 10 & & & & & 68 & 18 & 29 & & & \\
\hline & $\mathrm{m} 28$ & 76 & 62 & & & & & 68 & 18 & 29 & & & \\
\hline & m29 & 10 & 62 & & & & & 68 & 18 & 29 & & & \\
\hline & $\mathrm{m} 30$ & 76 & 10 & 62 & & & & & & & 13 & 25 & \\
\hline & m31 & 76 & 10 & 62 & & & & & & & 13 & 41 & \\
\hline & $\mathrm{m} 32$ & 76 & 10 & 62 & & & & & & & 25 & 41 & \\
\hline & m33 & 76 & 10 & & & & & & & & 13 & 25 & 41 \\
\hline & m34 & 76 & 62 & & & & & & & & 13 & 25 & 41 \\
\hline & m35 & 10 & 62 & & & & & & & & 13 & 25 & 41 \\
\hline & m36 & & & & 49 & 57 & 85 & 68 & 18 & & & & \\
\hline & m37 & & & & 49 & 57 & 85 & 68 & 29 & & & & \\
\hline & m38 & & & & 49 & 57 & 85 & 18 & 29 & & & & \\
\hline & m39 & & & & & 49 & 57 & 68 & 18 & 29 & & & \\
\hline & $\mathrm{m} 40$ & & & & & 49 & 85 & 68 & 18 & 29 & & & \\
\hline & $\mathrm{m} 41$ & & & & & 57 & 85 & 68 & 18 & 29 & & & \\
\hline & $\mathrm{m} 42$ & & & & 49 & 57 & 85 & & & & 13 & 25 & \\
\hline & $\mathrm{m} 43$ & & & & 49 & 57 & 85 & & & & 13 & 41 & \\
\hline & m44 & & & & 49 & 57 & 85 & & & & 25 & 41 & \\
\hline & m45 & & & & & 49 & 57 & & & & 13 & 25 & 41 \\
\hline & m46 & & & & & & 85 & & & & 13 & 25 & 41 \\
\hline & $\mathrm{m} 47$ & & & & & & 85 & & & & 13 & 25 & 41 \\
\hline & $\mathrm{m} 48$ & & & & & & & 68 & 18 & 29 & 13 & 25 & \\
\hline & $\mathrm{m} 49$ & & & & & & & 68 & 18 & 29 & 13 & 41 & \\
\hline & m50 & & & & & & & 68 & 18 & 29 & 25 & 41 & \\
\hline & m51 & & & & & & & 68 & 18 & & 13 & 25 & 41 \\
\hline & m52 & & & & & & & 68 & 29 & & 13 & 25 & 41 \\
\hline & m53 & & & & & & & 18 & 29 & & 13 & 25 & 41 \\
\hline Type C: & m54 & 76 & 10 & 62 & & 85 & & & 29 & & & & \\
\hline Mixing 3 & m55 & 76 & 10 & 62 & & & & & 68 & & & 41 & \\
\hline FMG & m56 & 76 & 10 & 62 & & 49 & & & & & & 25 & \\
\hline & m57 & & & & 49 & 57 & 85 & & 18 & & & 13 & \\
\hline & m58 & & 76 & & 49 & 57 & 85 & & 68 & & & & \\
\hline & m59 & & 10 & & 49 & 57 & 85 & & & & & 41 & \\
\hline & $\mathrm{m} 60$ & & 62 & & & & & 68 & 18 & 29 & & 25 & \\
\hline & m61 & & 76 & & & 57 & & 68 & 18 & 29 & & & \\
\hline & m62 & & & & & 85 & & 68 & 18 & 29 & & 13 & \\
\hline & m63 & & 10 & & & 49 & & & & & 13 & 25 & 41 \\
\hline & m64 & & 62 & & & & & & 29 & & 13 & 25 & 41 \\
\hline & m65 & & & & & 57 & & & 18 & & 13 & 25 & 41 \\
\hline
\end{tabular}


activity potential, we selected a total of 25 strains from bagasse and 20 strains from straw (45 strains) (Fig. S2 Supplementary information; identity of these 45 strains can be found in Table S1).

\section{Metabolic Diversity and Construction of Consortia}

In order to construct the MAMC, we first characterized the metabolic profile of the 45 selected strains using Biolog GEN III plates. A principal component analysis (PCA) performed with the data obtained after $72 \mathrm{~h}$ of incubation revealed distinct metabolic profiles that were not associated with the substrate type used for the enrichment (bagasse or straw) (Fig. 1). Most of the variation is explained by the first axis (35.66\%); however, variation is moderately explained by the second axis (17.36\%). We clustered the PCA results according to the four resulting sections in the plot (I, II, III, and IV denoted by different colors in Fig. 1), thus determining four distinct FMG. We noticed that according to the nature of the Biolog substrates (Table S2 supplementary information), functional metabolic groups III and IV have a preference for

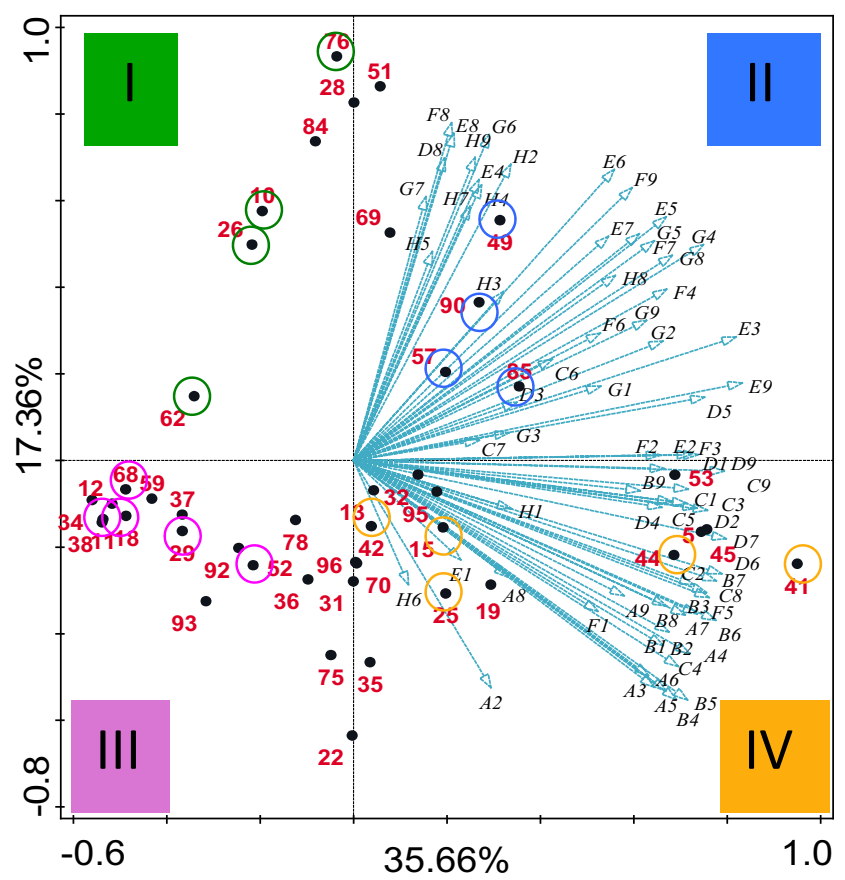

Fig. 1 Principal component analysis (PCA) of the individual bacterial stains, which led to their classification into four functional metabolic groups (FMG), according to the four sections in the plot (I, II, III, IV), as indicated by the different colors. The graph is based on the metabolic potential of the individual strains, as determined by measuring the area under the curve after $72 \mathrm{~h}$ of incubation of the 45 selected strains in Biolog GEN III plates. Blue vectors represent the different energy sources of Biolog GEN III plates. Bigger-colored circles indicate the 18 selected strains that were used to construct the MAMC and their respective FMG carbohydrate metabolism. Groups I and II, on the other hand, have a preference for amino acids and carboxylic acids.

A total of 18 representative strains from each of the FMG (see Table 2) were then used to construct five-species MAMC, thus creating a total of 65 synthetic communities by combining members of two, three, or all four functional metabolic groups (Table 1).

\section{Degradation of Lignocellulose Fractions and Correlation with Metabolic Potentials}

FTIR analysis performed on dried substrate obtained after consortia's growth depicts degradation of lignocellulose per lignocellulose's fraction (lignin, cellulose, and hemicellulose). Figure 2 shows the percentage of degradation of all 65 synthetic communities including three replicates containing all 18 strains per lignocellulosic fraction. From the results, we could distinguish that the synthetic communities type A (MAMC with four functional groups, $\mathrm{m} 2-\mathrm{m} 17$ ) degraded lignin effectively when compared to the degradation of hemicellulose and cellulose. On the other hand, type $\mathrm{C}$ synthetic communities (MAMC with three functional groups, m54-m65) have a better degradation of hemicellulose. A MDS (Fig. S3 Supplementary information) plotting mixes type A and type $\mathrm{C}$ shows a fair separation between these two types. Synthetic communities type B (MAMC with two functional groups, m18-m53), however, do not have a clear clustering since the degradation levels of all three lignocellulosic fractions were rather heterogeneous among communities (data not plotted). The synthetic community containing all 18 strains shows the highest degradation profile of all lignocellulosic fractions (52\% on average).

In order to measure the degree of functionality across the synthetic communities, and their potential effect on lignocellulose degradation, we used three FD measures: (i) FMG, which represents the number of functional groups in the synthetic community; (ii) $\mathrm{CN}$; and (iii) FAD, both of which are associated with the metabolic potential of the community. Our results revealed that both the FD and the lignocellulose component influenced the relationship (Fig. 3). FMG did not generate any significant pattern, although a positive relationship between lignin degradation and FMG was close to significant ( $p$ value $=0.061$ ), a positive tendency was also observed in $\mathrm{CN}$ results. A positive relationship was equally observed between the degradation of lignin ( $p$ value $=0.025)$ and FAD, although the explanatory power was very low $\left(R^{2}=0.0238\right)$. Conversely, higher metabolic diversity $(\mathrm{CN})$ had a negative and significant effect on the degradation of cellulose and negative tendency on the degradation of hemicellulose, showing here a similar pattern than those obtained with FMG and FAD. 
Table 2 Selected strains from each of the four functional metabolic groups

\begin{tabular}{|c|c|c|}
\hline $\begin{array}{l}\text { Functional } \\
\text { metabolic group }\end{array}$ & ID strain no. & Identification \\
\hline \multirow[t]{4}{*}{ I } & 76 & Cupriavidus pauculus partial 16S rRNA gene strain KPS201 \\
\hline & 10 & Pseudomonas plecoglossicida strain ICB-TAB78 16S ribosomal RNA gene \\
\hline & 26 & Alcaligenes sp. DA5 16S ribosomal RNA gene \\
\hline & 62 & Paracoccus sp. B160 16S ribosomal RNA gene \\
\hline \multirow[t]{4}{*}{ II } & 49 & Achromobacter sp. HBCD-1 16S ribosomal RNA gene \\
\hline & 90 & Devosia riboflavina strain HPG62 16S ribosomal RNA gene \\
\hline & 57 & Ochrobactrum sp. 71B2 $16 \mathrm{~S}$ ribosomal RNA gene \\
\hline & 85 & Sphingobacterium sp. Bt-34 16S ribosomal RNA gene \\
\hline \multirow[t]{5}{*}{ III } & 68 & Brevundimonas sp. R3 16S ribosomal RNA gene \\
\hline & 11 & Cellulosimicrobium sp. BAB-2381 16S ribosomal RNA gene \\
\hline & 29 & Chryseobacterium taiwanense strain DUCC3723 16S ribosomal RNA gene \\
\hline & 52 & Flavobacterium sp. WG1 partial 16S rRNA gene strain WG1 \\
\hline & 18 & Paenibacillus sp. PALXIL05 16S ribosomal RNA gene \\
\hline \multirow[t]{5}{*}{ IV } & 41 & Enterobacter aerogenes strain K_G_AN-5 16S ribosomal RNA gene \\
\hline & 44 & Pseudomonas sp. GT 2-02 16S ribosomal RNA gene \\
\hline & 25 & Microbacterium sp. UYFA68 16S ribosomal RNA gene \\
\hline & 15 & Bacillus nealsonii strain RTA5b2 16S ribosomal RNA gene \\
\hline & 13 & Stenotrophomonas maltophilia strain JN40 16S ribosomal RNA gene \\
\hline
\end{tabular}

\section{Identification of Potential Strains and the Best MAMC}

We identified a total of seven synthetic communities with an averaged degradation $>30 \%$ (maximum value $50.6 \%$ ) in all three lignocellulosic fractions: $\mathrm{m} 5, \mathrm{~m} 22, \mathrm{~m} 28, \mathrm{~m} 33, \mathrm{~m} 48$, $\mathrm{m} 53$, and $\mathrm{m} 61$. A RDA triplot using the degradation results data vs the combination of strains (as environmental data) revealed that the seven synthetic communities with relatively high degradation clustered together and shared specific strains (Fig. 4). Table 3 summarizes the degradation results of the seven synthetic communities.

The most effective lignocellulose-degrading strains common among the seven selected synthetic communities were Stenotrophomonas maltophilia strain JN40 (ID Number. 13), Paenibacillus sp. PALXIL05 (ID Number 18), Microbacterium sp. UYFA68 (ID Number 25), Chryseobacterium taiwanense
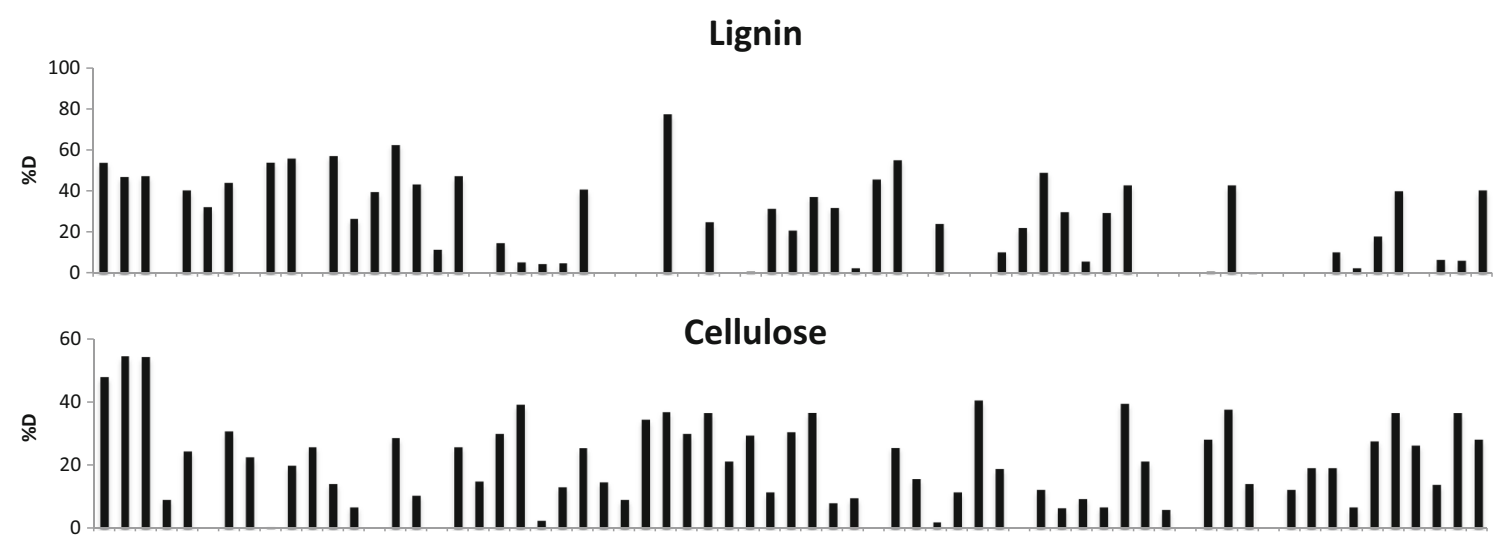

\author{
Hemicellulose
}

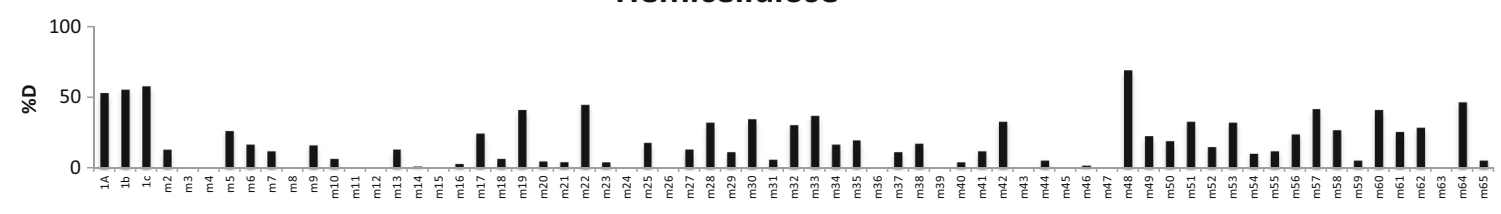

Fig. 2 Percentage of degradation $(\% \mathrm{D})$ of the three lignocellulosic fractions obtained with FTIR for each of the MAMC - synthetic communities of five species (m2-m65), taken from a pool of 18 selected lignocellulose degrading strains. The first three bars (1a-1c) represent the degradation potential of all 18 strains combined 

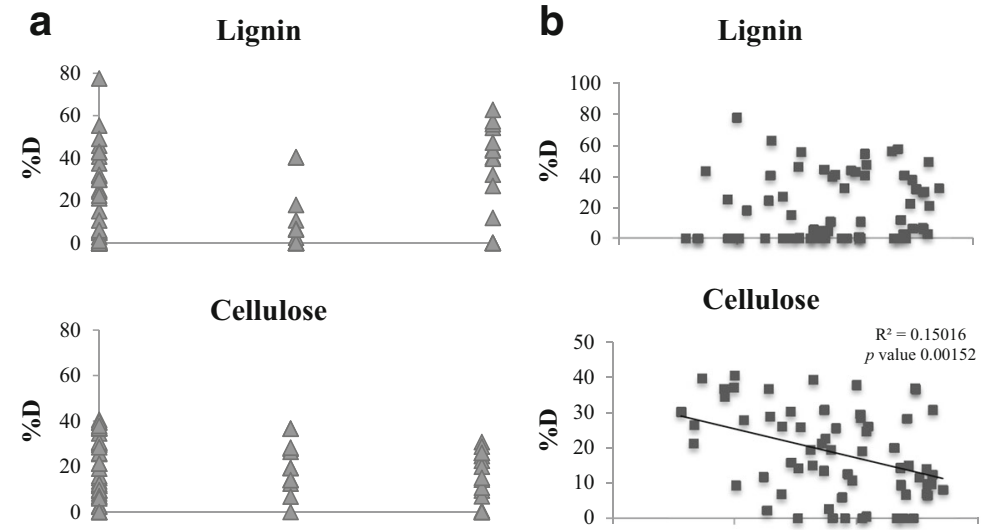

Hemicellulose

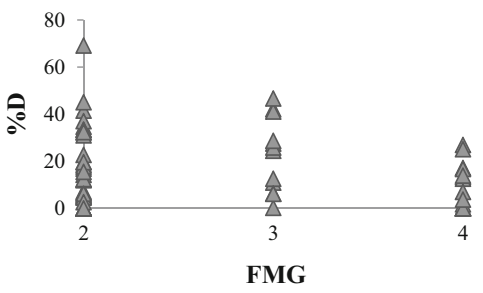

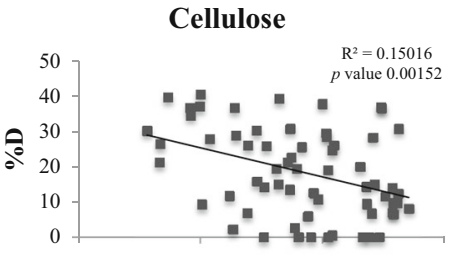

Hemicellulose

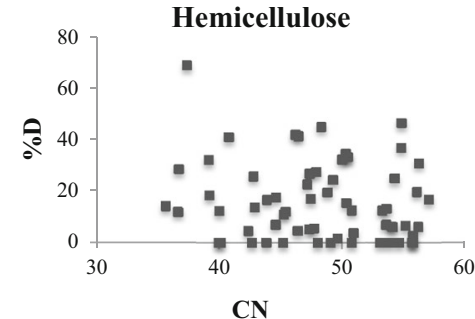

C

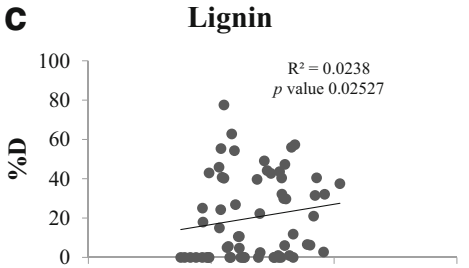

Cellulose

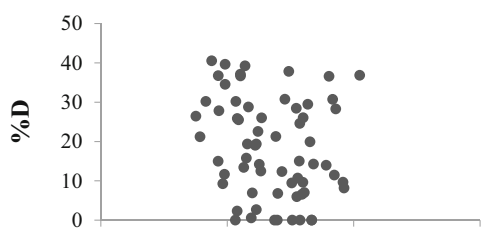

Hemicellulose

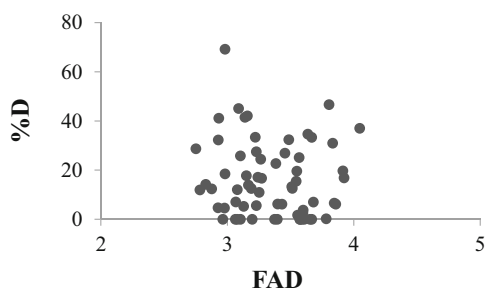

Fig. 3 Relationship between functional diversity measurements and the degradation potential of the microbial consortia in each of the lignocellulose fraction: lignin, cellulose, and hemicellulose. a Degradation $(\% \mathrm{D})$ as a function of the number of functional metabolic groups (FMG), defined according to the metabolic potential of the strains used in the used in the synthetic communities (see Fig. 1). b Degradation $(\% \mathrm{D})$ as a function of the community niche $(\mathrm{CN})$, which represents the

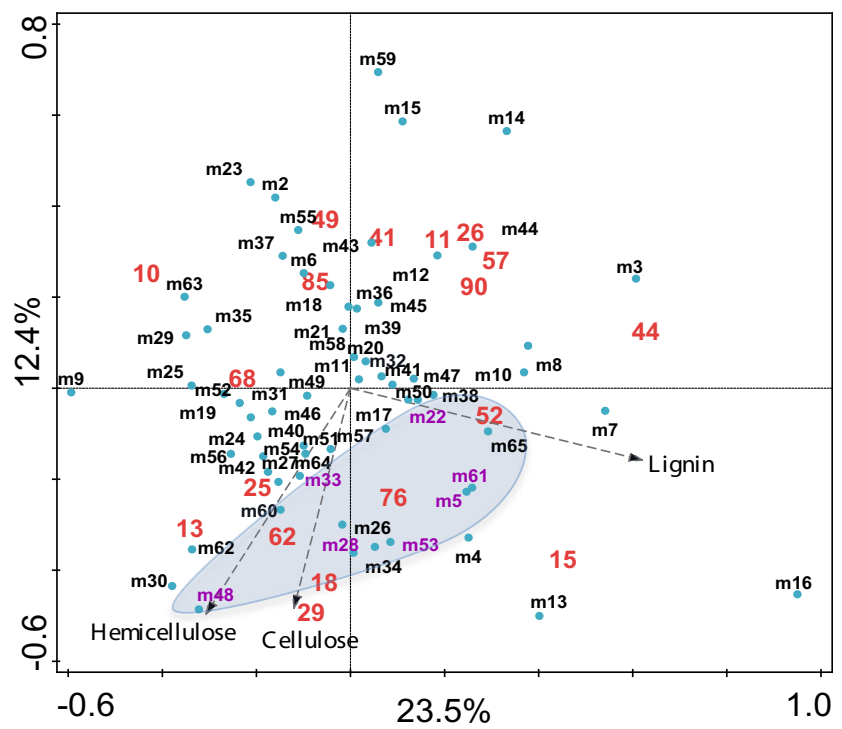

Fig. 4 Multivariate analyses depicting the degradation potential of all MAMC. Redundancy analyses (RDA) were performed using the degradation results on all three lignocellulose components of all synthetic communities (blue circles) as well as the individual strains (as environmental data, in red). Degradation activity of MAMC is based on the FITR method whereas that from individual strains is based on MUF analyzes. Arrows indicate the activity of each lignocellulose component. An ellipse highlights the synthetic communities with $>30 \%$ degradation potential, when compared to the total amount of lignocellulose available in the sugarcane bagasse or straw maximum metabolic potential of the mixture [9, 10]. $\mathbf{c}$ Degradation (\%D) as a function of the functional attribute diversity (FAD), calculated using the Biolog metabolic data from Biolog GEN III plates. Functional diversity measurements were calculated using the data generated by the metabolic potential of the individual strains. Only significant relationships are indicated

strain DUCC3723 (ID Number 29), Paracoccus sp. B160 (ID Number 62), Brevundimonas sp. R3 (ID Number. 68), Burkholderia sp. YX02 16S ribosomal RNA gene (ID Number 76). According to the degradation results. the highest degradation was observed in the synthetic community number 48 (degradation averaged of all lignocellulose fractions $50.6 \%$ ) containing strains with ID numbers 13, 18 25, 29. and 68. All the strains in this synthetic community belonged to the functional metabolic groups with preference for carbohydrate metabolism (groups III and IV).

Table 3 The seven synthetic communities with degradation results $\% \mathrm{D}$ $>30$ and their specific degradation for each one of the 3 lignicellulose components, lignin, cellulose and hemicellulose

\begin{tabular}{llll}
\hline & \multicolumn{2}{l}{ Degradation $(\% \mathrm{D})$} \\
\cline { 2 - 4 } Synthetic community & Lignin & Cellulose & Hemicellulose \\
\hline $\mathrm{m} 5$ & 44.21 & 30.74 & 26.93 \\
$\mathrm{~m} 22$ & 40.79 & 25.51 & 45.09 \\
$\mathrm{~m} 28$ & 25.08 & 36.73 & 32.28 \\
$\mathrm{~m} 33$ & 37.56 & 36.84 & 37.05 \\
$\mathrm{~m} 48$ & 43.01 & 39.65 & 69.18 \\
$\mathrm{~m} 53$ & 42.76 & 37.83 & 32.38 \\
$\mathrm{~m} 61$ & 40.32 & 36.67 & 25.81 \\
\hline
\end{tabular}




\section{Discussion}

In this study, we used a reductive-screening approach coupled with ecological strategies to obtain minimal active microbial consortia (MAMC) capable of effectively degrading lignocellulose, using bacterial strains obtained from environmental (soil) samples. Our reductive method included an enrichment experiment and the isolation of final community members. Furthermore, we used enzymatic and metabolic-profile assessments to determine the degradation potential and metabolic property of the isolated members of the community. Enzymatic assessments are rather simple, powerful, and quantitative approaches for screening purposes and have been effectively used in analyzing lignocellulose degradation capacities $[26,29,40]$. Here, we used this method for screening purposes, focusing only on enzymes from the GH family, which are related to hemicellulose and cellulose degradation. Furthermore, the use of metabolic profiling tools like Biolog was advantageous in depicting metabolic preferences of the initially selected strains, define functional metabolic groups, identify the metabolic preferences of the effective degrading bacteria, and reveal the relationship between functional diversity and degradation.

Using the metabolic and enzymatic characterization of the strains together with 16S rRNA gene sequencing, we further designed 65 MAMC (bacterial communities containing synthetic communities of only five microbial species) that were functionally diverse, i.e., displaying various levels of complementarity in terms of both carbon source utilization and enzymatic activities associated with the different lignocellulose components. These functionally complementary communities were then assessed for their capacity to degrade all three lignocellulose fractions (see Fig. S1(b) in the supplementary information for a schematic representation of the reductive screening approach). Our aim was to determine whether high functional diversity would lead to higher degradation potential, as predicted in community ecology theory [41], given that high functionality is expected to lead to higher number of (complementary) functions or ecological roles needed for an effective degradation.

Our results showed that the number of strains included in the consortia did not determine the effectivity of degradation since the results obtained with all 18 strains were comparable to the results obtained with several five-strain constructed synthetic communities (the synthetic community with the maximum averaged degradation reached up to $96.5 \%$ of the degradation rate when compared to the synthetic community containing all 18 strains). Other studies obtained similar degradation levels (between 40 and $50 \%$ average of all three lignocellulosic fractions $[28,29]$ with more than 20 different identified strains in the community). Here, we could identify a total of seven synthetic communities with optimal degradation levels. Results were mostly identity dependent, but there were positive and negative connections with functional diversity. We can then conclude that reducing the number of community members provides a better overview of functional diversity and ecological roles without affecting the degradation levels. In another notice, we found that higher functional diversity increased the degradation of the most complex substrate (lignin). Lignin's breakdown involves the release of a great variety of compounds including complex aromatic carboxylic acids, which might eventually converge into the citric acid cycle [42]. Our results suggest that the strains with the enzymatic machinery able to metabolize lignin metabolic products (aromatic carboxylic acids) might also have the enzymatic means to metabolize complex substrates like lignin.

Thus, the so-called division of labor together with diversity in our built consortia might be acting beneficially upon the potential of the community during lignin degradation. Our results showed that a higher functional diversity could expand and add complementarity for resource use among taxa in the context of bacterial community functioning [38]. On the other hand, the degradation levels of cellulose negatively correlated with the diversity measures based on overall carbon metabolism (FAD and $\mathrm{CN}$ ) whereas non-significant but negative trends were observed for hemicellulose. The enzymatic degradation of these substrates is accomplished via the collective action of multiple carbohydrate-active enzymes, typically acting together as a cocktail with complementary, synergistic activities and modes of action [6]. This negative relationship could suggest the presence of interspecific competition, which can be a stress factor for the community.

Interestingly, we found commonality in the species among the seven selected synthetic communities with the highest degradation levels, which are frequently reported as effective lignocellulose degraders. For example, Paracoccus sp. was found to be dominant after selection in lignin-amended lignocellulosic cultures; this suggested that Paracoccus sp. has an active role in lignin modification and depolymerization [2, 43]. On the other hand, Burkholderia sp. has been found to secrete specific enzymes capable of degrading plant cell wall components like cellulose, hemicellulose, lignin, and xylose $[44,45]$. Another study found specific genes coding for catalases and peroxidases, which might contribute to the lignin degrading ability of Burkholderia sp. [45]. Our RDA analysis showed a positive correlation of Burkholderia sp. with the degradation of all three lignocellulosic fractions.

Other genera included in the most effective synthetic communities were Stenotrophomonas maltophilia, Microbacterium sp., Paenibacillus sp., Chryseobacterium taiwanense, and Brevundimonas sp. The genus Stenotrophomonas occurs ubiquitously in nature and species like $S$. maltophilia are often found in the rhizosphere and inside many different plant species. Interestingly, Galai et al. [46] demonstrated laccase activity in Stenotrophomonas maltophilia, which was able to deconstruct lignin. Hence, Stenotrophomonas sp. has been found as excellent candidate for biotechnological applications. On the 
other hand, although there are very few reports of Microbacterium sp. strains with lignin degradation ability, Microbacterium species were found to be present in the gut of the wood-infesting termites [47]. There are also reports of strains of Microbacterium able to degrade polycyclic aromatic hydrocarbons [48-50].

Several strains of Paenibacillus sp. have been found to efficiently degrade lignocellulosic fractions [51], having effective lignolytic and cellulosytic enzymes [50, 52, 53]. On the other hand, the role of Chryseobacterium sp. in lignocellulose degradation is still unclear [30]; however, several species of Chryseobacterium have been isolated from lignocellulosic substrates and as part of the degradation processes of cellulose [54]. We found here (Fig. 3) a strong correlation of cellulose degradation with the presence of Paenibacillus sp. and Chryseobacterium sp. in the consortia. Moreover, the presence of Brevundimonas sp. (catalase producers) during lignocellulose degradation has been reported in lignin-amended soils [43] and in enrichment cultures of switchgrass and corn stover [26] and also has been found to be related to cellulose degradation [55].

Here, we were able to construct minimal effective consortia from environmental samples with optimal degradation levels as the ones found in consortia built with a higher number of community members. We found that a consortium containing Stenotrophomonas maltophilia, Paenibacillus sp., Microbacterium sp., Chryseobacterium taiwanense, and Brevundimonas sp. is an effective degrading synthetic community. Further work would be needed to understand the interactive relationship and metabolic pathways of these consortium partners. However, the screening method described here demonstrated to be a useful way to obtain minimal lignocellulose-degrading consortia from environmental samples that can be further developed to efficiently promote a sustainable way of lignocellulose breakdown for environmental or commercial purposes.

Acknowledgments This work was part of the Microbial Consortia for Biowaste Management-Life cycle analysis of novel strategies of bioconversion (MICROWASTE) project. We thank the Fundação de Amparo à Pesquisa do Estado de São Paulo (FAPESP, Brazil) and The Netherlands Organization for Scientific Research-— NWO for providing financial support (FAPESP-NWO No. 729004006.).

Funding This study was funded by FAPESP-NWO grant No.729004006.

\section{Compliance with Ethical Standards}

Conflict of Interest The authors declare that they have no conflict of interest.

Ethical Approval This article does not contain any studies with human participants or animals performed by any of the authors.

Open Access This article is distributed under the terms of the Creative Commons Attribution 4.0 International License (http:// creativecommons.org/licenses/by/4.0/), which permits unrestricted use, distribution, and reproduction in any medium, provided you give appropriate credit to the original author(s) and the source, provide a link to the Creative Commons license, and indicate if changes were made.

\section{References}

1. Bhatia L, Johri S, Ahmad R (2012) An economic and ecological perspective of ethanol production from renewable agro waste: a review. AMB Express 2:65

2. Lotfi G (2014) Lignin-degrading bacteria. J Agroaliment Process Technol. 20(1):64-68

3. Eiteman MA, Lee SA, Altman E (2008) A co-fermentation strategy to consume sugar mixtures effectively. J Biol Eng. 2:3

4. Lynd LR, Weimer PJ, Van Zyl WH, Pretorius IS (2002) Microbial cellulose utilization: fundamentals and biotechnology. Microbiol Molecul Biol Rev. 66:506-739

5. Szambelan K, Nowak J, Czarnecki Z (2004) Use of Zymomonas mobilis and Saccharomyces cerevisiae mixed with Kluyveromyces fragilis for improved ethanol production from Jerusalem artichoke tubers. Biotechnol Lett. 26:845-848

6. Cragg SM, Beckham GT, Bruce NC, Bugg TDH, Distel DL, Dupree P, Etxabe AG, Goodell BS, Jellison J, McGeehan JE, McQueen-Mason SJ, Schnorr K, Walton PH, Watts JEM, Zimmer M (2015) Lignocellulose degradation mechanisms across the Tree of Life. Curr Opin Chem Biol. 29:108-119

7. Bell T, Newman JA, Silverman BW, Turner SL, Lilley AK (2005) The contribution of species richness and composition to bacterial services. Nature 436:1157-1160

8. Wittebolle L, Marzorati M, Clement L, Balloi A, Daffonchio D, Heylen K, De Vos P, Verstraete W, Boon N (2009) Initial community evenness favours functionality under selective stress. Nature 458:623-626

9. Salles JF, Poly F, Schmid B, Le Roux X (2009) Community niche predicts the functioning of denitrifying bacterial assemblages. Ecology 90:3324-3332

10. Mallon CA, Poly F, Le Roux X, Marring I, van Elsas JD, Salles JF (2015) Resource pulses can alleviate the biodiversity-invasion relationship in soil microbial communities. Ecology 96:915-926

11. Schink B (2002) Synergistic interactions in the microbial world. Antonie Van Leeuwenhoek 81:257-261

12. Stolyar S, Van Dien S, Hillesland KL, Pinel N, Lie TJ, Leigh JA, Stahl DA (2007) Metabolic modeling of a mutualistic microbial community. Mol Syst Biol. 3:92

13. Hug LA, Beiko RG, Rowe AR, Richardson RE, Edwards EA (2012) Comparative metagenomics of three Dehalococcoides containing enrichment cultures: the role of the non-dechlorinating community. BMC Genomics 13:327

14. Brenner K, You L, Arnold FH (2008) Engineering microbial consortia: a new frontier in synthetic biology. Trends Biotechnol. 26(9):483-489

15. Mee MT, Fig HH (2012) Engineering ecosystems and synthetic ecologies. Mol BioSyst. 8(10):2470-2483

16. Feng Y, Yu Y, Fig X, Qu Y, Li D, He W, Kim B (2011) Degradation of raw corn stover powder (RCSP) by an enriched microbial consortium and its community structure. Bioresour Technol. 102(2):742-747

17. Okeke BC, Lu J (2011) Characterization of a defined cellulolytic and xylanolytic bacterial consortium for bioprocessing of cellulose and hemicelluloses. Appl Biochem Biotechnol. 163:869-881

18. Fig C, Dong D, Wang H, Müller K, Qin Y, Wang H, Wu W (2016) Metagenomic analysis of microbial consortia enriched from compost: new insights into the role of Actinobacteria in lignocellulose decomposition. Biotechnol Biofuels 9:22

19. Liang J, Peng X, Yin D, Li B, Fig D, Lin Y (2015) Screening of a microbial consortium for highly simultaneous degradation of lignocellulose and chlorophenols. Bioresour Technol. 190:381-387 
20. Jiménez DJ, Chaves-Moreno D, van Elsas JD (2015) Unveiling the metabolic potential of two soil-derived microbial consortia selected on wheat straw. Sci Rep. 5:13845

21. Colombo LT, de Oliveira MN, Carneiro DG, de Souza RA, Alvim MC, Dos Santos JC, da Silva CC, Vidigal PM, da Silveira WB, Passos FM (2016) Applying functional metagenomics to search for novel lignocellulosic enzymes in a microbial consortium derived from a thermophilic composting phase of sugarcane bagasse and cow manure. Antonie Van Leeuwenhoek 109(9):1217-1233

22. Maruthamuthu M, Jiménez DJ, Stevens P, van Elsas JD (2016) A multi-substrate approach for functional metagenomics-based screening for (hemi)cellulases in two wheat straw-degrading microbial consortia unveils novel thermoalkaliphilic enzymes. BMC Genomics 17:86. https://doi.org/10.1186/s12864-016-2404-0

23. Nelson CE, Gardner JG (2015) In-frame deletions allow functional characterization of complex cellulose degradation phenotypes in Cellvibrio japonicas. Appl Environ Microbiol. 81:175968-175975

24. Bernstein HC, Carlson RP Microbial consortia engineering for cellular factories: in vitro to in silico systems. Comput Struct Biotechnol J 3:e201210017

25. Xia Y, Ju F, Fang HHP, Zhang T (2013) Mining of novel thermostable cellulolytic genes from a thermophilic cellulose-degrading consortium by metagenomics. PLOS. https://doi.org/10.1371/ journal.pone.0053779

26. Jiménez DJ, de Lima Brossi MJ, Schückel J (2016) Characterization of three plant biomass-degrading microbial consortia by metagenomics- and metasecretomics-based approaches. Appl Microbiol Biotechnol. https://doi.org/10.1007/s00253-016-7713-3

27. Singh KM, Reddy B, Patel D, Patel AK, Parmar N, Patel A, Patel JB, Joshi CG (2014) High potential source for biomass degradation enzyme discovery and environmental aspects revealed through metagenomics of Indian buffalo rumen. Biomed Res Int 2014: 267189. https://doi.org/10.1155/2014/267189

28. Brossi MJL, Jiménez DJ, Cortes-Tolalpa L, van Elsas JD (2016) Soil-derived microbial consortia enriched with different plant biomass reveal distinct players acting in lignocellulose degradation. Microb Ecol. 71:616-627

29. Cortes-Tolalpa L, Jiménez DJ, de Lima Brossi MJ, Salles JF, van Elsas JD (2016) Different inocula produce distinctive microbial consortia with similar lignocellulose degradation capacity. Appl Microbiol Biotechnol. 100(17):7713-7725

30. Jiménez DJ, Dini-Andreote F, van Elsas JD (2014) Metataxonomic profiling and prediction of functional behaviour of wheat straw degrading microbial consortia. Biotechnol Biofuels 7:92

31. Versalovic J, Koeuth T, Lupski JR (1991) Distribution of repetitive DNA sequences in eubacteria and application to fingerprinting of bacterial genomes. Nucleic Acids Res. 19:6823-6831

32. Edwards U, Rogall T, Blocker H, Emde M, Bottger EC (1989) Isolation and direct complete nucleotide determination of entire genes - characterization of a gene coding for 16s-ribosomal RNA. Nucleic Acids Res. 17:7843-7853

33. Lane DJ (1991) 16S/23S rRNA sequencing. In: Stackebrandt E, Goodfellow M (eds) Nucleic acid techniques in bacterial systematics. John Wiley and Sons, Chichester, pp 115-175

34. Taketani RG, Franco NO, Rosado AS, van Elsas JD (2010) Microbial community response to a simulated hydrocarbon spill in mangrove sediments. J Microbiol. 48:7-15

35. ter Braak CJF, Šmilauer P (2012) Canoco reference manual and user's guide: software for ordination version 5.0. Microcomputer Power Ithaca

36. Adapa PK, Tabil LG, Schoenau GJ, Canam T, Dumonceaux T (2011) Quantitative analysis of lignocellulosic components of non-treated and steam exploded barley, canola, oat and wheat straw using
Fourier transform infrared spectroscopy. Res Creat Act. 107. http:// thekeep.eiu.edu/bio fac/107

37. Walker B, Kinzig A, Langridge J (1999) Plant attribute diversity, resilience, and ecosystem function: the nature and significance of dominant and minor species. Ecosystems 2:95-113

38. Salles JF, Le Roux X, Poly F (2012) Relating phylogenetic and functional diversity among Denitrifiers and quantifying their capacity to predict community functioning. Front Microbiol 3:209

39. Petchey OL, Gaston KJ (2002) Functional diversity (FD), species richness and community composition. Ecol Lett. 5:402-411

40. Kračun SK, Schückel J, Westereng B, Thygesen LG, Monrad RN, Eijsink VGH, Willats WGT (2015) A new generation of versatile chromogenic substrates for high-throughput analysis of biomassdegrading enzymes. Biotechnol Biofuels 8:70

41. Hooper DU, Chapin FS, Ewel JJ, Hector A, Inchausti P, Lavorel S, Lawton JH, Lodge DM, Loreau M, Naeem S, Schmid B, Setälä H, Symstad AJ, Vandermeer J, Wardle DA (2005) Effects of biodiversity on ecosystem functioning: a consensus of current knowledge. Ecol Monogr. 75:3-35

42. Fisher AB, Fong SS (2014) Lignin biodegradation and industrial implications. AIMS Bioeng 1(2):92-112

43. DeAngelis KM, Allgaier M, Chavarria Y, Fortney JL, Hugenholtz P, Simmons B, Sublette K, Silver WL, Hazen TC (2011a) Characterization of trapped lignin-degrading microbes in tropical forest soil. PLoS One 6:e19306

44. Coenye T, Vandamme P (2003) Diversity and significance of Burkholderia species occupying diverse ecological niches. Environ Microbiol. 5:719-729

45. Woo HL, Hazen TC, Simmons BA, DeAngelis KM (2014) Enzyme activities of aerobic lignocellulolytic bacteria isolated from wet tropical forest soils. Syst Appl Microbiol. 37:60-67

46 Galai S, Limam F, Marzouk MN (2009) A new Stenotrophomonas maltophilia strain producing laccase. Use in decolorization of synthetics dyes. Appl Biochem Biotechnol. 158(2):416-431

47 Wenzel M, Schönig I, Berchtold M, Kämpfer P, König H (2002) Aerobic and facultatively anaerobic cellulolytic bacteria from the gut of the termite Zootermopsis angusticollis. J Appl Microbiol. 92:32-40

48 Zhang HM, Kallimanis A, Koukkou AI, Drainas C (2004) Isolation and characterization of novel bacteria degrading polycyclic aromatic hydrocarbons from polluted Greek soils. Appl Microbiol Biotechnol. 65:124-131

49 Taylor CR, Hardiman EM, Ahmad M, Sainsbury PD, Norris PR, Bugg THD (2012) Isolation of bacterial strains able to metabolize lignin from screening of environmental samples. J Appl Environ Microbiol. https://doi.org/10.1111/j.1365-2672.2012.05352.x

50 Wang Y, Liu Q, Yan L, Gao Y, Wang Y, Wang W (2013) A novel lignin degradation bacterial consortium for efficient pulping. Bioresour Technol. 139:113-119. https://doi.org/10.1016/j.biortech.2013.04.033

51 Mathews SL, Grunden AM, Pawlak J (2016) Degradation of lignocellulose and lignin by Paenibacillus glucanolyticus. Int Biodeterior Biodegrad 110:79e86

52 Wang CM, Shyu CL, Ho SP, Chiou SH (2008) Characterization of a novel thermophilic, cellulose-degrading bacterium Paenibacillus sp. strain B39. Lett Appl Microbiol. 47:46-53

53 Mohanram S, Amat D, Choudhary J, Arora A, Nain L (2013) Novel perspectives for evolving enzyme cocktails for lignocellulose hydrolysis in biorefineries. Sustain Chem Processes 1:15

54 Maki ML, Idrees A, Leung KT, Qin W (2012) Newly isolated and characterized bacteria with great application potential for decomposition of lignocellulosic biomass. J Mol Microbiol Biotechnol. 22:156-166

55 Eichorst SA, Kuske CR (2012) Identification of celluloseresponsive bacterial and fungal communities in geographically and edaphically different soils by using stable isotope probing. Appl Environ Microbiol. 78(7):2316-2327 